\title{
Genomic regions influencing gene expression of the HMW glutenins in wheat
}

\author{
Eric W. Storlie · Robert J. Ihry · Leslie M. Baehr · Karissa A. Tieszen · Jonathan H. Engbers • \\ Jordan M. Anderson-Daniels · Elizabeth M. Davis · Anne G. Gilbertson · Niels R. Harden · Kristina A. Harris • \\ Amanda J. Johnson · Amy M. Kerkvleit - Matthew M. Moldan · Megan E. Bell · Michael K. Wanous
}

Received: 7 February 2008/Accepted: 16 September 2008/Published online: 7 October 2008

(c) The Author(s) 2008. This article is published with open access at Springerlink.com

\begin{abstract}
Bread wheat (Triticum aestivum L.) produces glutenin storage proteins in the endosperm. The HMW glutenins confer distinct viscoelastic properties to bread dough. The genetics of HMW glutenin proteins have been extensively studied, and information has accumulated about individual subunits, chromosomal locations and DNA sequences, but little is known about the regulators of the HMW glutenins. This investigation addressed the question of glutenin regulators. Expression of the glutenins was analyzed using QRT-PCR in ditelosomic (dt) Chinese Spring (CS) lines. Primers were designed for each of 4 CS
\end{abstract}

Communicated by B. Friebe.

E. W. Storlie - J. M. Anderson-Daniels .

A. G. Gilbertson · M. M. Moldan · M. K. Wanous ( $\square)$

Department of Biology, Augustana College,

Sioux Falls, SD 57197, USA

e-mail: mike.wanous@augie.edu

Present Address:

R. J. Ihry · K. A. Tieszen

Cell and Molecular Biology Program,

University of Wisconsin, Madison, WI 53706, USA

Present Address:

L. M. Baehr

Section of Neurobiology, Physiology, and Behavior,

University of California, Davis, CA 95616, USA

Present Address:

J. H. Engbers

Carver College of Medicine,

University of Iowa, Iowa, IA 52242-2600, USA

Present Address:

E. M. Davis

Sanford School of Medicine,

University of South Dakota, Vermillion, SD 57069, USA glutenin genes and a control, non-storage protein endosperm-specific gene Agp-L (ADP-glucose pyrophosphorylase). Each line represents CS wheat, lacking one chromosome arm. The effect of a missing arm could feasibly cause an increase, decrease or no change in expression. For each HMW glutenin, results indicated there were, on average, 8 chromosome arms with an up-regulatory effect and only one instance of a down-regulatory effect. There were significant correlations between orthologous and paralogous HMW glutenins for effects of chromosome groups B and D. Some or all the glutenin alleles shared regulatory loci on chromosome arms 2BS,

Present Address:

N. R. Harden

Mayo School of Graduate Medical Education,

Rochester, MN 55905, USA

Present Address:

K. A. Harris

Department of Nutritional Sciences,

Pennsylvania State University,

University Park, PA 16802, USA

Present Address:

A. J. Johnson

University of North Dakota School of Medicine \& Health

Sciences, Grand Forks, ND 58202, USA

Present Address:

A. M. Kerkvleit

Creighton University School of Medicine,

Omaha, NE 68131, USA

Present Address:

M. E. Bell

Biology Department, Bethel University,

St Paul, MN 55112-6999, USA 
7BS, 4DS, 5DS and 6DS, and Agp-L shared regulatory loci with glutenins on arms 7AS, 7BS, 2DS, 3DS, 4DS and 5DS. These results suggest a few chromosome arms contain putative regulatory genes affecting the expression of conserved cis elements of $4 \mathrm{HMW}$ glutenin and Agp-L genes in CS. Regulation by common genes implies the regulators have diverged little from the common wheat ancestor, and furthermore, some regulation may be shared by endosperm-specific-genes. Significant common regulators have practical implications.

\section{Introduction}

Wheat is an allohexaploid crop plant belonging to the grass (Poaceae) family. Hexaploid wheat consists of three genomes-A, B and D-produced by relatively recent polyploidization events. Each genome is homoeologous to the other genomes and consists of very similar orthologous loci. Gu et al. (2004) suggest the A and D genomes were derived from a common ancestor after separation of the $\mathrm{B}$ genome species. Subsequently, the A and B genomes were united when an A genome donor, T. urartu, crossed with an unknown B genome donor and produced T. turgidum. A couple hundred thousand years later T. turgidum crossed with a D genome donor, T. tauschii, and produced T. aestivum (Feldman et al. 1995).

Unusual cytological events resulted in duplicated genomes and loci, including paralogous genes residing on the same chromosomes as the original loci. Duplicated loci may facilitate the plasticity and evolution of an organism by retaining the original function of a gene while allowing its paralog and ortholog to take on modified or novel functions (see reviews by RA Veitia 2005; Adams and Wendel 2005).

Wheat grain consists of starch $(70 \%)$, protein $(11 \%)$, fatty acids (3\%), ash (2\%), and fiber (1.5\%) (Clydesdale 1994). Storage proteins, glutenins and gliadins, are synthesized in the endosperm during the grain-filling period; they comprise $90 \%$ of the protein in the wheat grain (Huebner and Wall 1974; Pike and MacRitchie 2004). High molecular weight (HMW) glutenin subunits each contribute about $2 \%$ of the total seed protein (Payne et al. 1984; Halford et al. 1992) and contribute the unique elastic properties and quality of bread dough.

The HMW glutenins are encoded by loci on homoeologous chromosomes 1L. There are two closely linked paralogous genes, designated Glu-1-1 and Glu-1-2, on chromosomes $1 \mathrm{AL}, 1 \mathrm{BL}$ and $1 \mathrm{DL}$, resulting from an ancestral duplication event. The glutenin genes probably evolved from a single ancestral gene (Kreis et al. 1985). The Glu-1-1 and Glu-1-2 orthologs share more DNA sequence homology than the paralogs (Allaby et al. 1999). The genes Glu-1-1 and Glu-1-2 encode X (high $\mathrm{M}_{\mathrm{r}}$ ) and $\mathrm{Y}$ (low $\mathrm{M}_{\mathrm{r}}$ ) glutenins, respectively. Two genes are inactive in many cultivars; Chinese Spring (cv) has two inactive genes on chromosome $1 \mathrm{AL}$. The HMW glutenin genes occupying each locus have been cloned and characterized from each homoeologous chromosome (see review by Shewry et al. 2003).

Allelic variation for the active genes translates to HMW glutenin subunit variation. The various subunits have been genetically characterized and correlated with quality characteristics (Payne 1987). Some subunit combinations have a more favorable effect on quality than other combinations. Qualitative factors contributing elastic properties of bread dough may be the regularly occurring $\beta$-turns of HMW glutenin polypeptides (Shewry et al. 1989) and tyrosine bonds (Tilley et al. 2001). A quantitative factor contributing to quality includes the proportion of HMW glutenins in relation to other endosperm components (Huebner and Wall 1974; Field et al. 1983; Lawrence et al. 1988). Rooke et al. (1999) analyzed flour from wheat transformed to produce a higher proportion of HMW glutenins; results indicated increased dough strength.

Expression of the HMW glutenin genes determines the content level of subunits. Kolster et al. (1993) analyzed the expression of alleles for Glu-Al, Glu-Bl and Glu-Dl from various donors in a common genetic background, nearisogenic lines, and results indicated the background did not affect subunit ratios. Genetic background did affect properties of bread quality in an experiment involving four doubled haploid wheat populations (Killerman and Zimmerman 2000). Kolster et al. (1993) suggest glutenin loci share a common regulatory mechanism. The suggestion of a common mechanism implies common cis and trans regulators control the expression of glutenin alleles relatively the same, regardless of the genotype. However, an investigation by Thiellement et al. (1986) suggested the duplicated glutenin genes do not share regulation.

The cis regions of the HMW glutenins are conserved $1,200 \mathrm{bp}$ upstream of the start codon and 200-400 bp downstream of the stop codon (Anderson et al. 1998). Lamacchia et al. (2001) indicate the promoter sequence of $1,191 \mathrm{bp}$ upstream of the start site regulates endospermspecific expression. The upstream promoter regions consist of elements common to grasses in Poideae, prominently including an endosperm box-a GCN4-like motif (GLM, $5^{\prime}$-ATGAG/CTCAT- $\left.3^{\prime}\right)$ and a prolamin box (5'TGTAAAG- $3^{\prime}$ ) (Forde et al. 1985)—a AACA motif (Diaz et al. 2002) and an enhancer element, more specific to HMW glutenins (Thomas and Flavell 1990). Anderson et al. (2002) characterized $5^{\prime}$ and $3^{\prime}$ DNA of HMW glutenins and identified potential matrix-attachment regions (MARs), transposable elements (MITEs), and 
retrotransposons (LTR and non-LTR) flanking respective glutenin genes. Proposed transcription factors recognize conserved elements of glutenin promoters: the storage protein activator (SPA), a seed-specific basic leucine zipper protein, bZIP (Albani et al. 1997), DNA binding with one finger (Dof) prolamin box binding factor (VicenteCarbajosa et al. 1997) and a MYB-protein, possibly interacting with the Dof protein (Diaz et al. 2002). Wheat and other cereals share cis motifs and trans domains for the regulation of storage and possibly other proteins.

Approaches to the identification and mapping of regulators have included genetic mapping using aneuploid wheat (Galili and Feldman 1985; Rogers et al. 1990; Wanous et al. 2003) and genetic linkage analysis using wheat populations (Guillaumie et al. 2004). Guillaumie et al. (2004) genetically mapped SPA to the long arm of chromosome 1B, and Ravel et al. (2006) mapped Dof to the long arm of chromosome 5. Wanous et al. (2003) analyzed HMW glutenin proteins in ditelosomic wheat lines, and results indicated genes on several chromosome arms affected the regulation of each glutenin.

Regulation of gene expression may feasibly occur at any point between transcription and protein functionality. Some investigations suggest regulation of HMW glutenins occurs primarily at the transcriptional level (Bartels and Thompson 1986; Sorensen et al. 1989).

One objective of this investigation was to analyze HMW glutenin gene expression in ditelosomic wheat lines and to correlate these results with a previous study on protein expression (Wanous et al. 2003) and to determine if glutenins are regulated mostly at the transcriptional or posttranscriptional levels. Another objective was to correlate expression of orthologous and paralogous glutenin genes affected by the chromosome groups for indications of common chromosomal locations of glutenin regulation. The glutenin genes provide a unique set of genes to study the regulatory effects of duplicated genes in a polyploid plant. Also, common locations may contain important regulatory alleles contributing content level variation for the HMW subunits. Manipulation of this variation with molecular biological tools may facilitate plant selections for favorable qualities (Rathmell et al. 2001). A control, non-storage protein, endosperm-specific gene, $A g p-L$, was included for an indication of the specificity-tissue or protein class_-of gene expression.

\section{Materials and methods}

Plant material

Sears (1954) produced ditelosomic (dt) Chinese Spring wheat lines. For chromosome arms where dt lines were available, dt endosperm was compared to euploid CS seeds ( 0 vs. 3 doses in the endosperm). For chromosome arms where $\mathrm{dt}$ lines were not available, tetrasomic endosperm was compared to euploid CS endosperm (6 vs. 3 doses). CS and related aneuploid lines were obtained from the University of Missouri-Columbia. Three replicates of each CS euploid and aneuploid line were grown in $6^{\prime \prime}$-diameter pots filled with commercial potting soil. The growth chamber conditions were set at $25^{\circ} \mathrm{C}, 14 \mathrm{~h}$ of light from incandescent and metal halide lamps. The soil was saturated with water once a day and $1.0 \mathrm{~g}$ of Osmocote (Maryville, OH, USA) fertilizer applied to each plant, every 4 weeks.

\section{Seed harvest and RNA extraction}

Seeds were harvested 15 days post-anthesis (dpa). The embryo and pericarp were removed and the endosperm was placed in a microfuge tube and immersed in liquid $\mathrm{N}$ and subsequently stored at $-70^{\circ} \mathrm{C}$, until further processing. The endosperm was ground with mortar and pestle, frozen with liquid N. RNA was extracted using a modified Trizol (Invitrogen; Carlsbad, CA, USA) protocol. The RNA sample was treated with DNase and purified with Qiagen RNeasy kit (Germany). Samples were quantified and protein contamination estimated using a Hitachi spectrophotometer (Japan) and separated on a $1 \%$ agarose gel for an evaluation of degradation. Samples were stored at $-20^{\circ} \mathrm{C}$ until analysis.

\section{Primer design}

HMW glutenin nucleotide sequences were acquired from GenBank and aligned using MegAlign software (DNA*, Madison, WI, USA). Primers were designed for each glutenin gene based on nucleotide differences that distinguished each glutenin from the other three.

Genome specific primer design for the large subunit of $A D P$-glucose pyrophosphorylase (Agp-L) was executed by creating primers from a consensus sequence of Agp- $L$ GenBank accessions X66080, AF244997, EF405961 and AF492644. The primers amplified a $649 \mathrm{bp}$ fragment from diploid wheat progenitors: Triticum monococcum (genome donor, A), Aegilops speltoides (genome donor, B), and Triticum tauschii (genome donor, D). Amplicons from the progenitors were then sequenced.

These sequences were aligned to compare sequences from each genome. The alignment revealed genome specific single-nucleotide-polymorphisms (SNPs) for each genome and provided the basis for the design of an A genome specific $A g p-L$ primer pair. An $A g p-L$ primer pair designed for the A genome was confirmed to be genome specific by comparing presence and absence of amplicons between euploid CS and Dt1AS. The absence of an amplicon in the Dt1AS sample suggests the specificity of 
primers and chromosome location of $A g p-L$ within the A genome, on the long arm of chromosome 1A. Primer sequences $\left(5^{\prime}-3^{\prime}\right)$ for the housekeeping gene, $18 S$ rRNA, control, Agp-L and HMW glutenins:

\author{
Agp- $L$ consensus: \\ Forward, AAGCGATGAAAGTGGACACC \\ Reverse, CTTCTTGCACTCCCTCCTTG \\ (amplicon size $=649 \mathrm{bp}$ ) \\ Agp-L (A-genome specific): \\ Forward, TATATTGCGTCGATGGGAG \\ Reverse, ATTCAAACTTTGGAGGCTG \\ (amplicon size $=229$ bp) \\ $18 S$ rRNA: \\ Forward, CTGGGAGGGGCGCATTTATTAGAT \\ Reverse, CCCGGCCCAAGGTCCAACT \\ (amplicon size $=463 \mathrm{bp}$ ) \\ Glu-B1-1: \\ Forward, TAAGCGCCTGGTCCTCTTTG \\ Reverse, CACCTGTTGGCATGCCTC \\ (amplicon size $=118$ bp) \\ Glu-B1-2: \\ Forward, CGACAACCCATACCATGTTAACAC \\ Reverse, GGGTTGCTGCACCTTTGCC \\ (amplicon size $=70 \mathrm{bp}$ ) \\ Glu-D1-1: \\ Forward, GTCGCGGGACAATACGAG \\ Reverse, GTGCAGGTATTCCCCAAAATATAC \\ (amplicon size $=118 \mathrm{bp}$ ) \\ Glu-D1-2: \\ Forward, GCCAAGTGCCGCTCCGTC \\ Reverse, TGGTCTCACCAGGGTAGAAGGATCC \\ (amplicon size $=102 \mathrm{bp}$ )
}

\section{QRT-PCR}

A quantitative RT-PCR method estimated Cycle threshold $\left(C_{\mathrm{t}}\right)$ values, using a Cepheid SmartCycler II thermocycler (Sunnyvale, CA, USA), from the endosperm RNA. The $C_{\mathrm{t}}$ values of HMW glutenin and $A g p-L$ genes were normalized with $C_{\mathrm{t}}$ values of $18 \mathrm{~S}$ rRNA. All samples were replicated three times. An expression ratio was calculated from the normalized control, euploid CS, and the normalized samples, ditelosomic or tetrasomic CS. Significance was determined with $P$-values $(P<0.05)$ estimated from the "Pair Wise Fixed Reallocation Randomization Test," derived from the "Relative Expression Software Tool” (Pfaffl et al. 2001).

Correlation analysis

SPSS 12.1 (Chicago, IL, USA) was used to estimate Spearman's correlations ( $r$ ) of expression between HMW glutenin alleles.

\section{Results}

Quantitative RT-PCR was used to analyze the HMW glutenin gene expression of CS euploid and ditelosomic wheat lines for an estimate of chromosomal locations having a significant effect. Expression of HMW glutenins and Agp-L genes was significantly affected by putative regulatory genes residing on several chromosome arms (Fig. 1, Table 1). Expression of the Agp-L and each HMW glutenin gene was up-regulated by putative genes on 5-13 chromosome arms (Fig. 1; Table 1). In only one instance, for Glu-D1-2, was expression down-regulated by a gene(s) on 1DS. This observation is consistent with previous studies indicating the expression of structural genes is commonly influenced by multiple dosage sensitive regulatory loci (Guo and Birchler 1994). Gene expression was most significantly affected by $1 \mathrm{AL}, 1 \mathrm{BL}$ and 1DL for Agp-L, Glu$B 1$ and $G l u-D 1$, respectively, where these loci reside. In a previous study, Wanous et al. (2003) showed chromosomes $1 \mathrm{BL}$ and 1DL had the most significant effects on Glu-BI and $G l u-D 1$ protein expression. The corresponding results of these studies and a preceding investigation by Greene (1983) suggest HMW glutenin RNA transcript levels predict the final protein concentration and confirm the consistency and reliability of ditelosomic genetic stocks for expression analysis.

The HMW glutenin genes shared effects of putative regulatory loci on groups $\mathrm{B}$ and $\mathrm{D}$, based on strong correlations of expression effects of group $B$ and $D$ chromosome arms. Chromosome arms 2BS, 7BS and 5DS had significant up-regulatory effects on each of the glutenin genes; chromosome arm 4DS had significant effects on all glutenins, except Glu-D1-1; chromosome arm 6DS had significant effects on the paralogous genes, Glu-Bl-1 and Glu-B1-2; chromosome arm 2DS had significant effects on the paralogous genes, Glu-Bl-1 and Glu-D1-2 (Fig. 1). Chromosome arms 7BS, 2DS, 4DS and 5DS also had upregulatory effects on the control gene, Agp-L (Fig. 1).

The regulation of orthologous and paralogous genes may be shared, independent or both, depending on the evolution of cis elements and trans factors. There were significant correlations between orthologous and paralogous HMW glutenins for effects of chromosome groups B and D (Table 2). The correlations include the effects of all chromosome arms of a chromosome group, except the glutenin locus on 1L (Table 2). A correlation between glutenin gene expression indicates significant correlations $(P<0.01)$ for all possible pairs of $G l u-B 1$ and $G l u-D l$ for effects of chromosome group B (Table 2; Fig. 1b). The orthologs Glu-B1-1:Glu-D1-1 and Glu-B1-2:Glu-D1-2 and the paralogs Glu-B1-1:Glu-B1-2, Glu-B1-1:Glu-D1-2, GluB1-2:Glu-D1-1 and Glu-D1-1:Glu-D1-2 shared effects of chromosome group B arms (Table 2). There was one 


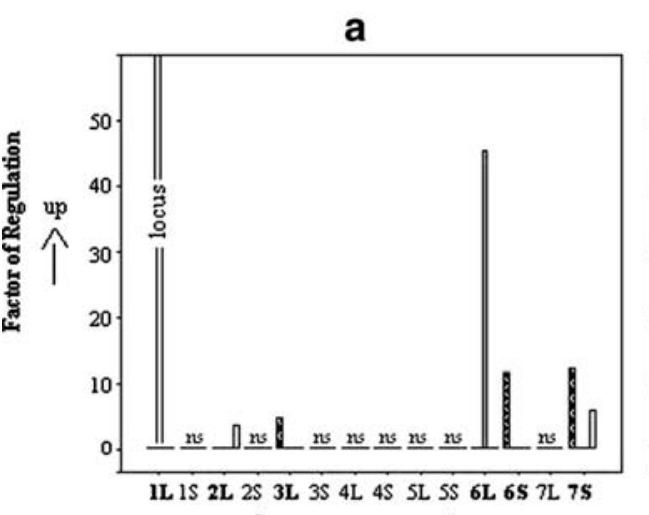

chromosome group A b

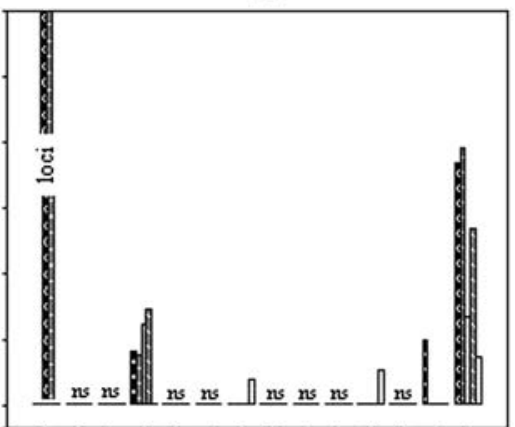

IL $1 S$ 2L $2 S 3 L$ SS $4 \mathrm{~L} 4 S$ SL SS $6 \mathrm{~L} 6 S 7 \mathrm{~L} 7 \mathrm{~S}$ chromosome group $B$
C

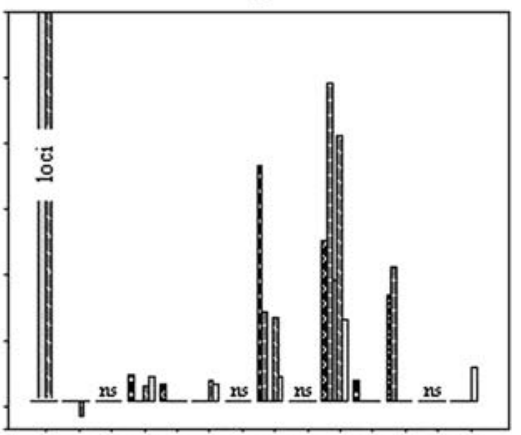

1L IS $2 \mathrm{~L} 2 \mathrm{~S}$ 3L $3 S 4 \mathrm{~L} 4 \mathrm{~S}$ SL $5 \$ 6 \mathrm{~L} 6 \mathrm{~S} 7 \mathrm{~L} 7 \mathrm{~S}$ chromosome group D

\section{Legend
Gu-B1-1 $\square$ Gu-B1-2 $\square$ Glu-D1-1 $\square$ Gu-D1-2 $\square$ Agp-L \\ ns no significant effect}

Fig. 1 Relative gene expression of HMW glutenins in ditelosomic wheat revealed chromosome arms where putative regulatory genes reside. Regulation was estimated as up or down regulated relative to euploid CS and a housekeeping gene, $18 \mathrm{~S}$ rRNA. Relative expression was estimated from an expression ratio $\left[2\left(2_{\text {target }}^{\left(C_{\text {contro }}-C_{\text {tsample }}\right)} /\right.\right.$ $\left.\left.2_{\text {reference }}^{\left(C_{\text {t }} \text { trample }\right.}\right)\right]$. Each graph depicts the effects of chromosome arms
1L-7S for each chromosome group-A (graph a) B (graph b) and D (graph c)—on expression of Glu-B1-1, Glu-B1-2, Glu-D1-1, and Glu-D1-2 and a control, non-storage protein gene, Agp-L. These genes are encoded on chromosome $1 \mathrm{~L}$, and the graphics for the effects of these chromosome arms represent up or down regulation of the loci

Table 1 Relative gene expression of HMW glutenins and Agp-L in ditelosomic wheat revealed chromosome arms where putative regulatory genes reside

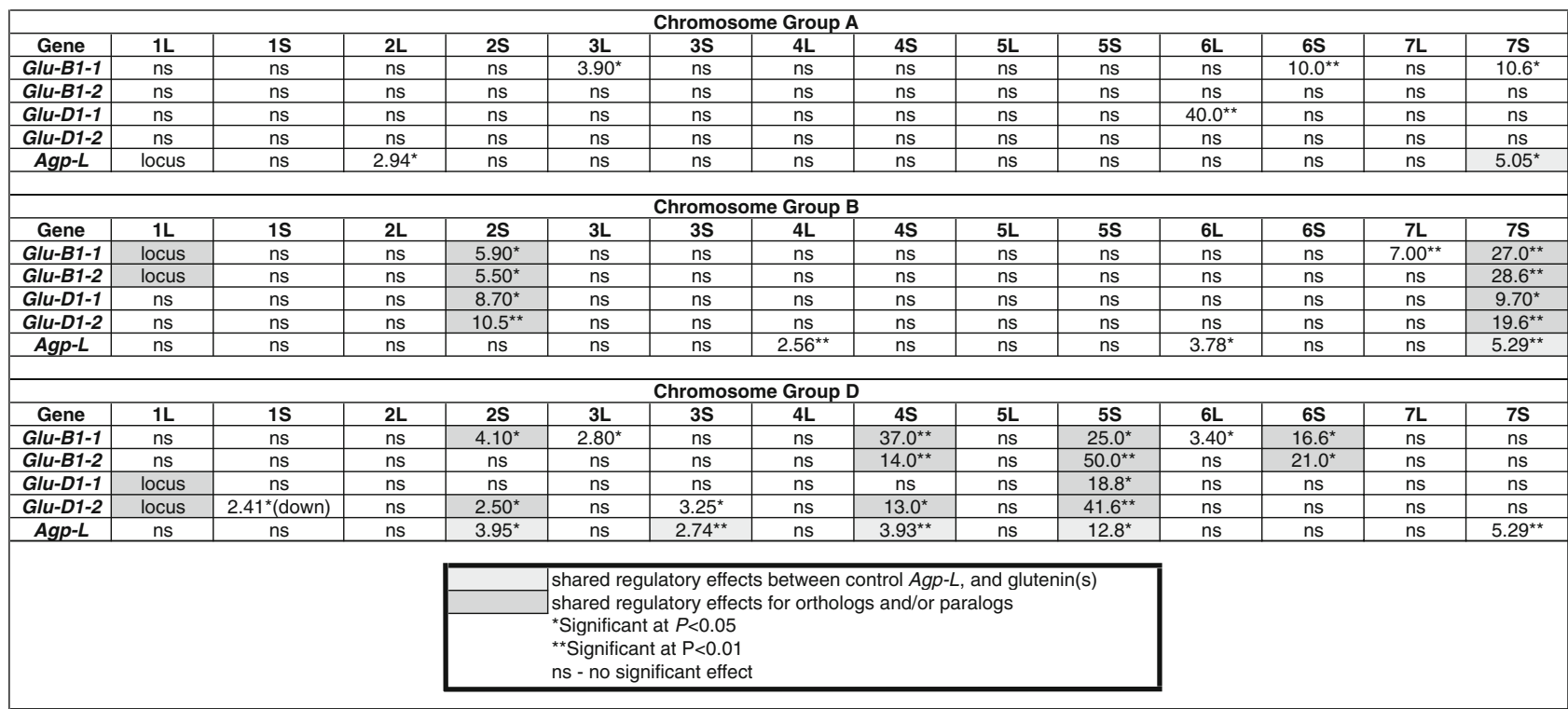

The HMW glutenin, Agp-L and $18 S$ rRNA genes are depicted in the first column to the left and are repeated in three groups, one for each chromosome-A, B and D. Significant regulatory effects of a chromosome arm are depicted as a factor of increased or decreased expression caused by a putative regulator residing on the chromosome arm. Significant regulatory effects on paralogs or orthologs and shared by $A g p-L$ are highlighted in gray. Regulation was up or down relative to euploid CS and a housekeeping gene, 18S rRNA. Relative expression was estimated from an expression ratio $\left[2_{\text {target }}^{\left(C_{\text {tcontrol }}-C_{\text {tsample }}\right)} / 2_{\text {reference }}^{\left(C_{\text {tontrol }}-C_{\text {tsample }}\right)}\right]$ 
Table 2 Bivariate correlations for the expression of HMW glutenin (Glu-B1-1, Glu-B1-2, Glu-D1-1 and Glu-D1-2) and Agp-L gene expression in CS wheat

\begin{tabular}{|c|c|c|c|c|c|c|c|c|c|c|c|c|c|c|c|c|}
\hline Gene & & Glu-B1-1 & Glu-B1-2 & Glu-D1-1 & Glu-D1-2 & Agp $-L$ & Glu-B1-1 & Glu-B1-2 & Glu-D1-1 & Glu-D1-2 & Agp-L & Glu-B1-1 & Glu-B1-2 & Glu-D1-1 & Glu-D1-2 & Agp-L \\
\hline & Chr. Group & A & A & A & $\mathbf{A}$ & A & B & $B$ & B & B & B & D & D & D & D & D \\
\hline Glu-B1-1 & $\mathbf{A}$ & & 0.00 & -0.14 & 0.00 & 0.39 & 0.27 & 0.39 & 0.39 & 0.39 & 0.27 & 0.10 & 0.14 & -0.14 & -0.20 & 0.08 \\
\hline Glu-B1-2 & $\mathbf{A}$ & & & & 0.00 & 0.00 & 0.00 & 0.00 & 0.00 & 0.00 & 0.00 & 0.00 & 0.00 & 0.00 & 0.00 & 0.00 \\
\hline Glu-D1-1 & A & & & & & -0.11 & -0.14 & -0.11 & -0.11 & -0.11 & 0.52 & 0.19 & -0.14 & -0.07 & -0.12 & -0.20 \\
\hline & & & & & & & & & & & & & & & & \\
\hline Glu-D1-2 & A & & & & & & 0.00 & 0.00 & 0.00 & 0.00 & 0.00 & 0.00 & 0.00 & 0.00 & 0.00 & 0.00 \\
\hline & & & & & & & & & & & & & & & & \\
\hline Agp-L & A & & & & & & 0.39 & 0.50 & 0.50 & 0.50 & 0.39 & -0.33 & -0.21 & -0.11 & -0.17 & 0.21 \\
\hline Glu-B1-1 & B & & & & & & & $0.78^{* *}$ & $0.78^{\star \star}$ & $0.78^{\star *}$ & 0.27 & -0.16 & -0.26 & -0.14 & -0.01 & 0.38 \\
\hline & & & & & & & & & & & & & & & & \\
\hline Glu-B1-2 & B & & & & & & & & $1.00^{* *}$ & $1.00^{* \star}$ & 0.39 & 0.00 & -0.21 & -0.11 & 0.09 & 0.59 \\
\hline & & & & & & & & & & & & & & & & \\
\hline Glu-D1-1 & B & & & & & & & & & $1.00^{* *}$ & 0.39 & 0.00 & -0.20 & -0.11 & 0.09 & 0.59 \\
\hline & & & & & & & & & & & & & & & & \\
\hline Glu-D1-2 & B & & & & & & & & & & 0.39 & 0.00 & -0.20 & -0.11 & 0.09 & 0.59 \\
\hline & & & & & & & & & & & & & & & & \\
\hline Agp-L & B & & & & & & & & & & & -0.16 & -0.26 & -0.14 & -0.22 & 0.08 \\
\hline & & & & & & & & & & & & & & & & \\
\hline Glu-B1-1 & D & & & & & & & & & & & & $0.77^{\star \star}$ & 0.42 & 0.59 & 0.41 \\
\hline & & & & \multirow{4}{*}{\multicolumn{2}{|c|}{$\begin{array}{l}\text { correlation of expr } \\
\text { orthologous } \\
\text { paralogous } \\
\text { with control, Agp-L }\end{array}$}} & ssion: & & & & & & & & & & \\
\hline Glu-B1-2 & D & & & & & & & & & & & & & 0.62 & 0.53 & 0.39 \\
\hline & & & & & & & & & & & & & & & & \\
\hline Glu-D1-1 & D & & & & & & & & & & & & & & 0.52 & 0.52 \\
\hline Glu-D1-2 & D & & & \multirow{2}{*}{\multicolumn{4}{|c|}{${ }^{* *}$ Significant at $p<0.01$}} & & & & & & & & & $0.75^{\star \star *}$ \\
\hline & & & & & & & & & & & & & & & & \\
\hline Agp-L & D & & & & & & & & & & & & & & & \\
\hline
\end{tabular}

Expression as depicted in Fig. 1 and Table 1 (except data for chromosomes $1 \mathrm{~L}$ because the structural gene effects overwhelm the detection of some correlations of expression on other chromosome arms) was correlated in all possible combinations. Significant correlations are depicted in bold with asterisks. Expression ratios for each gene were correlated with each other in all possible combinations using Spearman's correlation. The expression data for each glutenin and Agp-L gene analyzed for all chromosome arms of a chromosome group-A, B and D—were included in each correlation. Significant correlations are highlighted in bold with backgrounds of white or gray

significant correlation $(P<0.01)$ for effects of chromosome group D on the paralogs Glu-B1-1 and Glu-B1-2 and on the glutenin gene, Glu-Dl-2, and the control gene, Agp$L$ (Table 2; Fig. 1c). There were no significant correlations for any gene pairs for effects of chromosome group A or for effects of homoeologous chromosome groups-A:B, $\mathrm{A}: \mathrm{D}$ or $\mathrm{B}: \mathrm{D}$ - though there were instances of shared expression effects of 2BS:2DS, 3AL:3DL, 6AL:6DL, 6AS:6DS and 7AS:7BS on some glutenin genes (Table 1), and there were some instances of individual, non-shared effects of chromosome arms-3AL, 7BL, 1DS, 3DL and 3DS—on single glutenin genes (Table 1).

\section{Discussion}

The HMW glutenin gene expression on chromosomes 1BL and 1DL corresponds with the protein expression results (Wanous et al. 2003), confirming the methods of protein and mRNA quantification. There were no overall correlations $(P>0.05)$ between glutenin genes and proteins for effects of respective chromosome groups on expression; chromosome arms of each chromosome group uniquely affected gene and protein regulation. The difference between the putative regulatory genes for protein and gene expression are summarized for up and down regulation: glutenin protein expression was up-regulated by putative trans regulators on 10 chromosome arms and down-regulated by regulators on 5 chromosome arms, on average (protein data from Wanous et al. 2003); glutenin genes were up-regulated by putative trans regulators on 8 , on average, and down-regulated by 1 , only, chromosome $\operatorname{arm}(\mathrm{s})$. However, about $29 \%$ of the positive effects seen at the protein level (Wanous et al. 2003) also showed positive effects on transcript abundance in this study. These interactions include the positive effects of 2DS on Glu-Bl-1 and Glu-D1-2; 2BS on Glu-B1-2, Glu-D1-1 and Glu-D1-2; 5DS on Glu-B1-1 and Glu-D1-1; 6DS on Glu-B1-2; 6DL on Glu-B1-1; 6AL on Glu-D1-1; and 7BS on Glu-D1-2. These differences suggest HMW glutenin regulation occurs at various points between gene transcription and protein function; the HMW glutenin proteins probably undergo translational and post-translational control.

Ancient and relatively recent cytological events were responsible for the duplication of HMW glutenin genes resulting in paralogs and orthologs. One objective of this study was to compare the expression patterns of orthologous and paralogous HMW glutenin genes for implications of shared regulatory elements. Results indicate cis and trans elements on chromosome groups B and D are shared 
between paralogous and orthologous glutenins. There is no indication from this data that one duplication type was more conserved. Previous investigations have shown the orthologs are more highly conserved than the paralogs (Allaby et al. 1999; Anderson et al. 2002). Correlations between all combinations of glutenins and the control genes suggest chromosome group $\mathrm{B}$ has a common regulatory effect on glutenin paralogs and orthologs but not the control; group D had a common effect on Glu-B1-1:GluB1-2 and Glu-D1-2:Agp-L. The same chromosome group, rather than homoeologs, was involved with common regulatory effects; trans elements of one chromosome group had similar effects on the cis elements of several paralogs, orthologs and between one glutenin and Agp-L. Duplication of trans regulators on homoelogous chromosome arms was suggested for five chromosome arms-2S, 3L, 6L, 6S and $7 \mathrm{~S}$ - but shared regulation between homoeologs was minimal.

It has been hypothesized that aneuploid conditions may reduce vigor of an organism due to an imbalance of gene products expressed from the affected chromosome. Guo and Birchler (1994) conclude transcript levels for most genes maintained a constant level regardless of the chromosome dosage; regulatory genes were probably involved with the compensation of transcript levels of structural genes affected by chromosome dosage, increase or decrease. Guo and Birchler (1994) suggest the imbalance is a result of an altered regulatory system. In maize, Guo and Birchler (1994) identified 4 chromosomal regions with significant regulatory effects on the storage protein, Zein and 8 chromosomal regions with effects on Shrunken 1, Sucrose Synthase. The many regulators are part of a strict spatial and temporal development of specialized tissues. For example, Glu-Bl1 and $A g p-L$ were differentially expressed in the root compared with the grain tissue (Laudencia-Chingcuanco et al. 2006).

A conserved promoter sequence (Anderson et al. 1998) elicited a common response to putative regulators on certain chromosome arms; in many instances, putative regulators had an up-regulatory effect on an ancestral cis element retained by each orthologous and paralogous glutenin and Agp-L. Potential cis elements include the endosperm box, an AACA motif and an enhancer element (Forde et al. 1985; Thomas and Flavell 1990; Diaz et al. 2002). The common effect on the retained cis elements was initiated by putative trans factors residing on chromosomes 2BS, 7BS, 4DS, 5DS and 6DS. Potential transcription factors include the SPA, a seed-specific basic leucine zipper protein, $b Z I P$, Dof prolamin box binding factor and a MYB-protein, possibly interacting with the Dof protein (Albani et al. 1997; Vicente-Carbajosa et al. 1997; Diaz et al. 2002). Trans factors on the homoeologous chromosome arms did not produce the same effects (though there are other, minor examples in Table 1-2BS:2DS, 3AL:3DL, 6AL:6DL, 6AS:6DS and 7AS:7BS - of possible shared homoeologous trans regulators). After polyploidization, most orthologous trans factors may have been silenced due to an inefficient redundancy (see review by Wolfe 2001). Kashkush et al. (2002) analyzed $F_{1}$ intergeneric Triticeae hybrids for alterations of gene structure and expression: results indicate genetic and epigenetic changes occurred at the $F_{1}$ stage, causing gene loss, silencing and activation. Gu et al. (2004) elucidated possible mechanisms of gene silencing in wheat and concluded retrotransposon insertions were a primary cause. After gene duplication events, wheat has retained the functionality of some genes and silenced others. Anderson et al. (2002) identified transposable element and retrotransposon sequences in the flanking regions of HMW glutenins and suggest these were a mechanism of sequence divergence after the initial duplication event. The HMW glutenin and Agp-L genes have revealed shared regulation of duplicated and tissuespecific genes: hypothetically, cis regulatory elements of the glutenin paralogs, orthologs and Agp-L share the recognition of trans regulators on several chromosome arms (Fig. 1; Table 1).

Results of this investigation suggest a few chromosome arms contain regulatory genes affecting expression of 4 HMW glutenin and Agp-L genes. Regulation by common genes implies the work involved with identification of important regulatory genes is simplified and worthwhile. Notably, putative genes on chromosomes 2BS, 7BS and 5DS controlled a significant level of regulation for each paralogous and orthologous glutenin gene. Putative genes on 4DS and 6DS controlled regulation for some paralogous and orthologous glutenins. Genes controlling expression may be identified in a mapping study that isolates regions of these chromosome arms conferring variation for glutenin protein. Previous investigations have identified the chromosomal location of storage protein regulators (Guillaumie et al. 2004; Prasad et al. 1999; Colas des Frances and Thiellement 1985). Guillaumie et al. (2004) mapped SPA to chromosome group 1L, the same chromosomal arm as the HMW glutenin structural genes. Perretant et al. (2000) mapped bread-making quality and identified QTLs for dough strength on chromosomes 5D, $1 \mathrm{~A}$ and 3B. Ravel et al. (2006) mapped Dof on chromosome 5L. Putative regulator(s) on chromosome 5DS were important for expression of all the HMW glutenins. The isolated regions may reveal candidate regulatory genes in the bin maps available at GrainGenes. Candidate genes and molecular markers may be used in breeding programs to improve the glutenin protein and, possibly, starch composition of wheat. 
Evolutionary duplication events produced novel Triticum species with multiple loci of HMW glutenin genes. These genes have been well characterized and provide a unique opportunity to investigate gene expression effects of a polyploid genome on orthologous and paralogous genes. Hypothetically, there are several potential cis and trans regulatory adaptations, and this study provides insight into an example of how a polyploid plant conserved cis regulatory elements of active orthologs and paralogs (two previously investigated inactive glutenins on $1 \mathrm{AL}$ were caused by a relatively recent genetic or epigenetic alteration) and silenced redundant trans factors on most homoeologous chromosome arms. There were a few instances of independent regulators affecting the expression of one glutenin gene, and in most instances, putative regulators from one chromosome arm were shared by orthologs and paralogs. Shared regulation between glutenins and other endosperm-specific genes-Agp-L-residing on different genomes- $\mathrm{B}$ or $\mathrm{D}$-implies the cis and trans regulators did not diverge extensively from the common ancestor through the evolution and development of modern wheat.

Acknowledgments EWS, RJI, LMB, KAT, AGG, AJJ, MMM, and MKW were supported by NIH grant 2 P20 RR016479 from the INBRE Program of the National Center for Research Resources. LMB, JHE, JMA, EMD, and KAH were supported by the National Research Initiative of the USDA CSREES, grant 2003-35301-13216. NRH and AMK were supported by NSF EPSCoR grant 0091948 and by the State of South Dakota. RJI was supported by the NASA South Dakota Space Grant Consortium. We thank the reviewers for their comments.

Open Access This article is distributed under the terms of the Creative Commons Attribution Noncommercial License which permits any noncommercial use, distribution, and reproduction in any medium, provided the original author(s) and source are credited.

\section{References}

Adams KL, Wendel JF (2005) Polyploidy and genome evolution in plants. Curr Opin Plant Biol 8:135-141

Allaby RG, Banerjee M, Brown TA (1999) Evolution of the high molecular weight glutenin loci of the A, B, D, and G genomes of wheat. Genome 42:296-307

Albani D, Hammond-Kosack MCU, Smith C, Conlan S, Colot V, Holdsworth M, Bevan MW (1997) The wheat transcriptional activator SPA: a seed-specific bZIP protein that recognizes the GCN4-like motif in the bifactorial endosperm box of prolamin genes. Plant Cell 9:171-184

Anderson OD, Abraham-Pierce FA, Tam A (1998) Conservation in wheat high-molecular-weight glutenin gene promoter sequences: comparisons among loci and among alleles of the Glu-B1-1 locus. Theor Appl Genet 96:568-576

Anderson OD, Larka L, Christoffers KF, McCue KF, Gustafson JP (2002) Comparison of orthologous and paralogous DNA flanking the wheat high molecular weight glutenin genes: sequence conservation and divergence, transposon distribution, and matrix-attachment regions. Genome 45:367-380
Bartels D, Thompson RD (1986) Synthesis of messenger-RNAs coding for abundant endosperm proteins during wheat grain development. Plant Sci 46:117-125

Clydesdale FM (1994) Optimizing the diet with whole grains. Crit Rev Food Sci Nutr 34:453-471

Colas des Frances C, Thiellement H (1985) Chromosomal localization of structural genes and regulators in wheat by $2 \mathrm{D}$ electrophoresis of ditelosomic lines. Theor Appl Genet 71:31-38

Diaz I, Vicente-Carbajosa J, Abraham Z, Martinez M, Isabel-La Moneda I, Carbonero P (2002) The GAMYB protein from barley interacts with the DOF transcription factor BPBF and activates endosperm-specific genes during seed development. Plant J 29:453-464

Feldman M, Lupton FGH, Miller TE (1995) Wheats. In: Smart J, Simmonds NW (eds) Evolution of crops, 3rd edn. Longman Scientific, London, pp 184-192

Field JM, Shewry PR, Miflin BJ (1983) Solubilization and characterization of wheat gluten proteins: correlations between the amounts of aggregated proteins and baking quality. J Sci Food Agric 34:370-377

Forde BG, Heyworth A, Pywell J, Kreis M (1985) Nucleotide sequence of a $B 1$-hordein gene and the identification of possible upstream regulatory elements in endosperm storage protein genes from barley, wheat and maize. Nucl Acids Res 13:73277339

Galili G, Feldman M (1985) Genetic control of endosperm proteins in wheat. Theor Appl Genet 69:583-589

Greene FC (1983) Expression of storage protein genes in developing wheat (Triticum aestivum L.) seeds. Plant Phys 71:40-46

Gu YQ, Coleman-Derr D, Kong X, Anderson OD (2004) Rapid genome evolution revealed by comparative sequence analysis of orthologous regions from four Triticeae genomes. Plant Phys 135:459-470

Guillaumie S, Charmet G, Linossier L, Torney V, Robert N, Ravel C (2004) Colocation between a gene encoding the bZip factor SPA and an eQTL for a high-molecular-weight glutenin subunit in wheat (Triticum aestivum). Genome 47:705-713

Guo M, Birchler JA (1994) Trans-acting dosage effects on the expression of model gene systems in maize aneuploids. Science 266:1999-2002

Halford NG, Field JM, Blair H, Urwin P, Moore K, Robert L, Thompson R, Flavell RB, Tatham AS, Shewry PR (1992) Analysis of HMW glutenin subunits encoded by chromosome 1A of bread wheat (Triticum aestivum L.) indicates quantitative effects on grain quality. Theor Appl Genet 83:373-378

Huebner FR, Wall JS (1974) Fractionation and quantitative differences of glutenin from wheat varieties varying in baking quality. Cereal Chem 53:258-268

Kashkush K, Feldman M, Levy AA (2002) Gene loss, silencing and activation in a newly synthesized wheat allotetraploid. Genetics 160:1651-1659

Killerman B, Zimmerman G (2000) Relationship between allelic variation of Glu-1, Glu-3 and Gli-1 prolamin loci and baking quality in doubled haploid wheat populations. In: Shewry PR, Tatham AS (eds) Wheat Gluten. Royal Society of Chemistry, Cambridge, pp 66-70

Kolster P, Krechting CF, Van Gelder WMJ (1993) Expression of individual HMW glutenin subunit genes of wheat (Triticum aestivum L.) in relation to differences in the number and type of homoeologous subunits and differences in genetic background. Theor Appl Genet 87:209-216

Kreis M, Ford BG, Rahman S, Miflin BJ, Shewry PR (1985) Molecular evolution of the seed storage proteins of barley, rye and wheat. J Mol Biol 183:499-502

Lamacchia C, Shewry PR, Fonzo ND, Forsyth JL, Harris N, Lazzeri PA, Napier JA, Halford NG, Barcelo P (2001) Endosperm- 
specific activity of a storage protein gene promoter in transgenic wheat seed. J Exp Bot 52:243-250

Laudencia-Chingcuanco DL, Stamova BS, Lazo GR, Cui X, Anderson OD (2006) Analysis of the wheat endosperm transcriptome. J Appl Genet 47:287-302

Lawrence GJ, MacRitchie F, Wrighley CW (1988) Dough and baking quality of wheat lines deficient in glutenin subunits controlled by the Glu-A1, Glu-B1 and Glu-D1 loci. J Cereal Sci 7:109-112

Payne PI (1987) Genetics of wheat storage proteins and the effect of allelic variation on bread-making quality. Ann Rev Plant Phys 38:141-153

Payne PI, Jackson EA, Holt LM, Law CN (1984) Genetic linkage between endosperm storage protein genes on each of the short arms of chromosome 1A and 1B in wheat. Theor Appl Genet 67:235-243

Perretant MR, Cadalen T, Charmet G, Sourdille P, Nicolas P, Boeuf C, Tixier MH, Branlard G, Bernard S, Bernard M (2000) QTL analysis of bread-making quality in wheat using a doubled haploid population. Theor Appl Genet 100:1167-1175

Pfaffl MW, Horgan GW, Dempfle L (2001) Relative expression software tool (REST) for group-wise comparison and statistical analysis of relative expression results in realtime PCR. Nucleic Acids Res 30:1-10

Pike PR, MacRitchie R (2004) Protein composition and quality of some new hard white winter wheats. Crop Sci 44:173-176

Prasad M, Varshney RK, Kumar A, Balyan HS, Sharma PC, Edwards KJ, Singh H, Dhaliwal HS, Roy JK, Gupta PK (1999) A microsatellite marker associated with a QTL for grain protein content on chromosome arm 2DL of bread wheat. Theor Appl Genet 99:341-345

Rathmell WG, Wrigley CW, Batey IL, Howes N, Sharp PJ, Kilian A (2001) Selection of breeders lines for wheat quality: Australian innovations. In: Cauvin SP, Salmon SS, Young LS (eds) Using cereal science and technology for the benefit of the consumer. Woodhead Publishing, Cambridge, pp 24-28

Ravel C, Nagy IJ, Martre P, Sourdille P, Dardevet M, Balfourier F, Pont C, Giancola S, Praud S, Charmet G (2006) Single nucleotide polymorphism, genetic mapping, and expression of genes coding for the DOF wheat prolamin-box binding factor. Funct Integr Genomics 6:310-321

Rogers WJ, Rickatson JM, Sayers EJ, Law CN (1990) Dosage effects of chromosomes of homoeologous groups 1 and 6 upon bread- making quality in hexaploid wheat. Theor Appl Genet 80:281287

Rooke L, Bekes F, Fido R, Barro F, Gras P, Tatham AS, Barcelo P, Lazzeri P, Shewry PR (1999) Overexpression of a gluten protein in transgenic wheat results in greatly increased dough strength. J Cereal Sci 30:115-120

Sears ER (1954) The aneuploids of common wheat. Mo Agric Exp Stn Res Bull, 572

Shewry PR, Halford NG, Tatham AS (1989) The high molecular weight subunits of wheat, barley and rye: genetics, molecular biology, chemistry and the role in wheat gluten structure and functionality. In: Miflin BJ (ed) Oxford surveys of plant molecular and cell biology, vol 6. Oxford University Press, Oxford, pp 163-219

Shewry PR, Halford NG, Lafiandra D (2003) Genetics of wheat gluten proteins. Adv Genet 49:111-184

Sorensen MB, Cameron-Mills V, Brandt A (1989) Transcriptional and post-transcriptional regulation of gene expression in developing barley endosperm. Mol Gen Genet 217:195-201

Thomas MS, Flavell RB (1990) Identification of an enhancer element for the endosperm-specific expression of high molecular weight glutenin. Plant Cell 2:1171-1180

Thiellement H, Bahrman N, Colas des Frances C (1986) Regulatory effects of homoeologous chromosome arms on wheat proteins at two developmental stages. Theor Appl Genet 73:246-251

Tilley KA, Benjamin RE, Bagorogoza KE, Okot-Kober BM, Prakash O, Kwen H (2001) Tyrosine cross-links: molecular basis of gluten structure and function. J Agric Food Chem 49:2627-2632

Veitia RA (2005) Paralogs in polyploids: one for all and all for one? Plant Cell 17:4-11

Vicente-Carbajosa J, Moose SP, Parsons RL, Schmidt RJ (1997) A maize Zinc-finger protein binds the prolamin box in Zein gene promoters and interacts with the basic leucine zipper transcriptional activator Opaque2. Proc. Natl Acad Sci USA 94:76857690

Wanous MK, Munkvold JD, Kruse JD, Brachman EE, Klawiter MA, Fuehrer KJ (2003) Identification of chromosome arms influencing expression of the HMW glutenins in wheat. Theor Appl Genet 106:213-220

Wolfe KH (2001) Yesterday's polyploids and the mystery of diploidization. Nat Rev Genet 2:333-341 